Document downloaded from:

http://hdl.handle.net/10251/136516

This paper must be cited as:

Jimenez-Meneses, P.; Bañuls Polo, M.; Puchades, R.; Maquieira Catala, A. (2019). Novel and rapid activation of polyvinylidene fluoride membranes by UV light. Reactive and Functional Polymers. 140:56-61. https://doi.org/10.1016/j.reactfunctpolym.2019.04.012

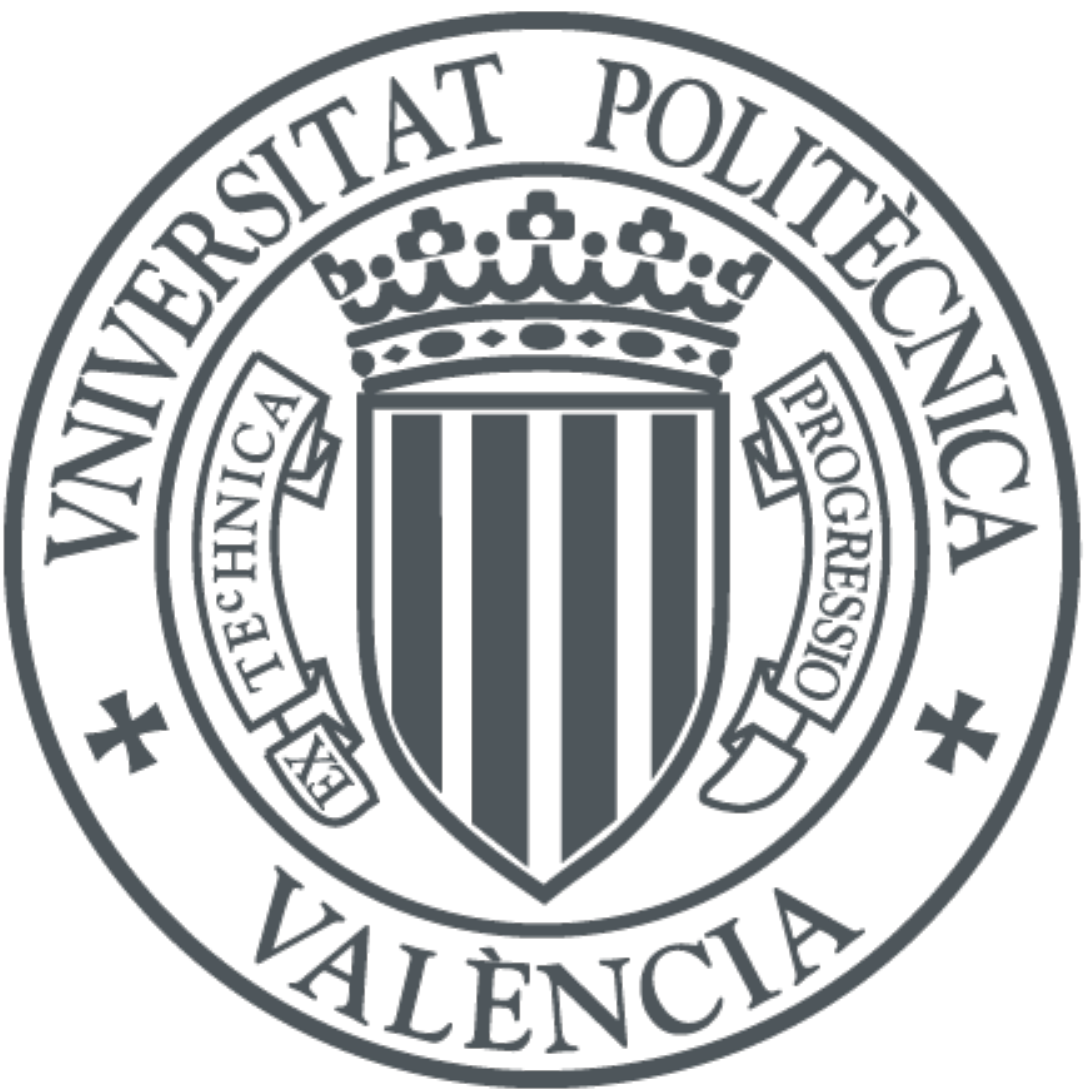

The final publication is available at

https://doi.org/10.1016/j.reactfunctpolym.2019.04.012

Copyright Elsevier

Additional Information 


\title{
Novel and rapid activation of polyvinylidene fluoride membranes by UV light
}

\author{
Pilar Jiménez-Meneses, María-José Bañuls* ${ }^{*}$ Rosa Puchades, Ángel Maquieira \\ Instituto Interuniversitario de Investigación de Reconocimiento Molecular y Desarrollo \\ Tecnológico (IDM) \\ Universitat Politècnica de València, Universitat de València, \\ Camino de Vera s/n, 46022 Valencia, Spain. E-mail: mbpolo@upvnet.upv.es
}

\begin{abstract}
Polyvinylidene fluoride (PVDF) membranes have become essential because of their huge applicability to the industry; however, they still present some limitations. This study focuses on the modification of PVDF membrane properties such as hydrophobicity, wettability, and functionality. To obtain a stable grafting, the surface of the membrane is hydroxylated using UV light at $254 \mathrm{~nm}$, followed by covalent immobilization of (3aminopropyl)triethoxysilane (APTES) and vinyltriethoxysilane (VTES). The physicochemical and morphological properties of modified and raw PVDF membranes were analyzed by spectroscopy, microscopy, and goniometry. Finally, nucleic acid microarray technology results showed that PVDF and PVDF-VTES membranes had probe immobilization densities of 5 and $11 \mathrm{pmol} / \mathrm{cm}^{2}$ and hybridization limits of detection of 1 and $5 \mathrm{nM}$, respectively.
\end{abstract}

Keywords

PVDF membranes

Hydroxylation

Silanization

Photoinduced immobilization

Nucleic acid microarrays 


\section{Introduction}

PVDF membranes have been extensively applied to industrial processes and scientific research because of their mechanical, chemical, and thermal resistance properties, and their simple fabrication in mass scale [1,2]. PVDF is a semi-crystalline fluoropolymer that exists in four forms, and it is made of $-\mathrm{CH}_{2} \mathrm{CF}_{2}-$ units. It has three types of bonds, namely, $\mathrm{C}-\mathrm{C}, \mathrm{C}-\mathrm{H}$, and $\mathrm{C}-\mathrm{F}$, whose energies are 88,106 , and $111 \mathrm{kcal} / \mathrm{mol}$, respectively $[1]$.

Because of the strong hydrophobic interactions exerted by the PVDF membrane, organic matter such as proteins accumulates into the pores, thereby lowering the filtration performance [3-5]; therefore, the antifouling properties of the membrane need to be improved. This can be achieved by increasing the membrane hydrophilicity and protein adsorption and reducing energy consumption in separation processes $[1,2,6]$. In addition, enhancement of the membrane hydrophobicity increases the wetting resistance, which is an important property addressed in different ways [2,7-10].

Therefore, change in the PVDF membrane composition to improve its applicability and performance by addition of hydrophilic or hydrophobic compounds is still under development. The main methodologies used thus far in the reformulation of PVDF membranes are surface modification by coating or grafting and blending modification by the addition of hydrophilic or amphiphilic polymers or inorganic nanoparticles in the fabrication process $[1,2,11-13]$. In this work, the membrane was modified by organosilane chemistry to achieve a covalent grafting. Hence, addition of hydroxyl groups onto the membrane surface by hydroxylation is necessary. Effective methodologies are necessary for hydroxylation, as most polymeric membranes such as PVDF do not present this functional group in their structure. 
For a long time, hydroxylation by alkaline treatment using concentrated sodium hydroxide has been extensively performed to increase the resistivity of the membrane to harsh environments by generating hydroxyl groups over the surface $[14,15]$. However, membrane degradation by dehydrofluorination, as a secondary effect, has been broadly reported $[1,10,14,16]$. Alternative methods as piranha-based process [17] and plasma treatment [18,19] have been described, which show different limitations [10], and therefore, it is necessary to develop new methodologies to carry out PVDF membrane modification.

In this work, although direct activation of the PVDF membranes by UV light is difficult because of the good UV photo-irradiation resistance of the PVDF membrane [20], activation using methanol and a powerful UV lamp has been accomplished. This approach is easy and rapid for the generation of oxygen-based reactive motifs on the structure, and it is also cleaner, simpler, and milder than the previous reported methods $[14,15,17-19]$. Through this methodology, hydroxyl groups are formed onto the surface, without affecting the bulk properties of the membrane. Thus far, UV light has been extensively applied by our group as a powerful tool for the hydroxylation of silicon-based surfaces [21-23]. Hence, silanization was easily performed later, which allowed the docking of the membranes with different organosilanes. Functionalization of PVDFbased materials by organosilane chemistry is scarcely reported in the literature, and the available studies have always used the difficult activation processes mentioned above (alkaline, plasma, and piranha treatments); therefore, this field has to be explored $[9,10,24]$.

Another interesting application of PVDF-based materials relies on the microarray technology. Since the advent of this technology in the 1990s, PVDF substrates have been widely used for the fabrication of protein microarrays. As mentioned before, PVDF 
materials demonstrate efficient hydrophobic interactions; hence, passive binding of proteins and other biomolecules to the substrate occurs, thereby displaying limited immobilization densities and high background signals [25-30]. For that, as a proof of concept, raw PVDF membrane and those functionalized with vinyltriethoxysilane (PVDF-VTES) were used for the development of covalently anchored nucleic acid microarrays. The covalent immobilization of thiolated probes allows a rapid, stable, and orientated bonding, hence increasing the amount of active receptors over the surface. For the tethering of the thiolated probes to PVDF membranes, thiol-ene and fluor-thiol photocoupling reactions were applied. Thiol-ene reaction has been extensively applied in the preparation of high-density microarrays, taking the advantages of the click chemistry reactions [22,31-33]. For that, the surface functionalization of the PVDF membranes was carried out to generate active alkene groups on it. For raw PVDF membranes, fluor-thiol reaction was employed. This promising reaction has been recently proposed by our group and allows the direct covalent bonding of thiolated probes to C-F motifs over the surface [21]. Finally, detection capacity of both membrane types was compared to that obtained in a previous work by performing hybridization assays [21].

Hence, in this study, we demonstrate an easy, rapid, and effective method to graft PVDF membranes with different functional groups by using microarray technology applications.

\section{Experimental}

\subsection{Chemicals, reagents, and buffers}

Immobilon-P PVDF membranes with $0.45 \mu \mathrm{m}$ nominal pore size were obtained from Merck Millipore (Spain). Ninhydrin, (3-aminopropyl)triethoxysilane (APTES), and vinyltriethoxysilane (VTES) were purchased from Sigma-Aldrich (Spain). Toluene and methanol were acquired from Scharlau (Spain). It is to be noted that all the chemicals 
were handled following the corresponding material safety data sheets. All chemicals were used without further purification.

Milli-Q water with a resistivity above $18 \mathrm{~m} \Omega$ was used to prepare aqueous solutions. All the buffers employed, phosphate-buffered saline (PBS1x, $0.008 \mathrm{M}$ sodium phosphate dibasic, $0.002 \mathrm{M}$ sodium phosphate monobasic, $0.137 \mathrm{M}$ sodium chloride, $0.003 \mathrm{M}$ potassium chloride, $\mathrm{pH} 7.5$ ), PBS-T (PBS1x containing 0.05\% Tween 20), saline sodium citrate (SSC1x, 0.15 M sodium chloride, $0.02 \mathrm{M}$ sodium citrate, $\mathrm{pH}$ 7), and SSC-T (SSC1x comprising $0.5 \%$ Tween 20), were filtered through a $0.22 \mu \mathrm{m}$ pore size nitrocellulose membrane from Whatman GmbH (Germany), before use.

The oligonucleotides listed in Table 1 were acquired from Eurofins Genomics (Germany). The quality and concentration of DNA were determined by measuring optical density at 260/280 nm using a NanoDrop ND 1000 spectrophotometer from Thermo Fisher Scientific (USA).

2.2. Instrumental methods

Water contact angle (WCA) measurements were made with Attension Theta Lite from Biolin Scientific (Sweden), and images were processed with version 3.1 of One Attension software from Biolin Scientific (Sweden). The measurements were made in quintuplicate at room temperature with a volume drop of $2 \mu \mathrm{L}$ employing $18 \mathrm{~m} \Omega$ water quality. Microarray printing was carried out with a low-volume noncontact dispensing system (AD 1500 model, Biodot, USA). Irradiation at $254 \mathrm{~nm}$ was carried out using a UV-Ozone Surface Cleaner, UVOH $150 \mathrm{Lab}$, from FHR (Germany), while irradiation at $365 \mathrm{~nm}$ was performed with a mercury capillary lamp from Jelight Irvine (USA), $6 \mathrm{~mW} / \mathrm{cm}^{2}$, placed at a fixed distance $(0.5 \mathrm{~cm})$. 
The fluorescence signal of the spots was recorded with a homemade surface fluorescence reader (SFR) having a high sensitive charge-coupled device camera, Retiga EXi from QImaging Inc., (Canada), with light emitting diodes, Toshiba TLOH157P, as the light source [34]. For microarray image analysis and subsequent quantification, GenePix Pro 4.0 software from Molecular Devices, Inc. (USA), was employed.

X-ray photoelectron spectra were documented with a Sage 150 spectrophotometer from SPECS Surface Nano Analysis GmbH (Germany). Nonmonochromatic Al Ka radiation $(1486.6 \mathrm{eV})$ operating at $30 \mathrm{eV}$ constant pass energy for elemental specific energy binding analysis was used as an X-ray source. Vacuum in the spectrometer chamber was $9 \times 10^{-9}$ $\mathrm{hPa}$, and the sample area examined was $1 \mathrm{~mm}^{2}$. Attenuated total reflectance infrared spectra were recorded using a Bruker Tensor 27 FT-IR system coupled to a Platinum ATR accessory from Bruker (Germany). Atomic force microscopy (AFM) measurements were carried out with a Veeco model Dimension 3100 Nanoman from Veeco Metrology (USA) at a tapping mode of $300 \mathrm{kHh}$. Imaging was performed in AC mode in air using OMCLAC240 silicon cantilevers from Olympus Corporation (Japan). The AFM images were taken at room temperature under ambient conditions, using tips from Nano World with a radius of $8 \mathrm{~nm}$. All images were processed with NanoScope software.

\subsection{Derivatization of membranes}

Immobilon-P PVDF membranes were cut into $2 \times 1 \mathrm{~cm}$ pieces, activated with methanol for 15 seconds, irradiated with a UV-Ozone surface cleaner for 10 minutes at $254 \mathrm{~nm}$, and immersed again in methanol for 15 seconds. To modify the surface properties, the PVDF membranes were then immersed into a $2 \% \mathrm{v} / \mathrm{v}$ solution of the corresponding silane in toluene at $40{ }^{\circ} \mathrm{C}$ for 1 hour. Then, the samples were withdrawn from the silane solution, 
washed several times with toluene and isopropanol, and air-dried. Finally, substrates functionalized with (3-aminopropyl)triethoxysilane (PVDF-APTES) were baked for 1 hour at $60{ }^{\circ} \mathrm{C}$, whereas those functionalized with vinyltriethoxysilane (PVDF-VTES) were baked for 1 hour at $80^{\circ} \mathrm{C}$. To corroborate the presence of primary amines in PVDFAPTES substrates, the ninhydrin test was performed. To carry out this selective test, a small piece of PVDF-APTES was immersed in $1.5 \mathrm{~mL}$ of ethanol. Then, $30 \mu \mathrm{L}$ of fresh ninhydrin solution in ethanol at $1 \%$ was added to the membrane. Finally, the mixture was heated for 5 minutes at $100{ }^{\circ} \mathrm{C}$. A piece of raw PVDF membrane underwent the same process to serve as a negative control.

2.4.Microarray of nucleic acid probes by covalent anchoring

Covalent anchoring of thiolated oligonucleotides onto raw PVDF and PVDF-VTES membranes by UV light at $365 \mathrm{~nm}$ was assessed. PVDF-VTES membranes were silanized as described above. Thus, $40 \mathrm{~nL}$ of Probe $1^{*}$, bearing Cy5 fluorophore in the 3' position and SH motif in the 5' position (Table 1), prepared in 1 and $2 \mu \mathrm{M} 1 \mathrm{X}$ PBS was spotted onto both surfaces to create the microarray (four spots per concentration). When the drops were dried, the PVDF membranes were exposed to UV light at $365 \mathrm{~nm}$ for 5 minutes to achieve immobilization. Afterwards, membranes were thoroughly rinsed with PBS-T for 10 minutes and air-dried, and fluorescence was measured using the SFR. Pictures of the membranes after irradiation as well as before and after washing were analyzed using GenePix software. Immobilization densities were calculated by comparing the fluorescence intensities before and after washing. For negative controls, two experiments were performed: first, adsorption of Probe $1 *$ was verified, and hence, the irradiation step 
was skipped; second, printing of a non-thiolated probe bearing a fluorophore in the 5' position (Target $1 *$ ) was performed, followed by irradiation later.

\subsection{Hybridization Assays}

To study the sensitivity of the modified and nonmodified surfaces, recognition assays were performed with raw PVDF and PVDF-VTES membranes. For that, PVDF-VTES membranes were functionalized as described above. Then, a thiolated oligonucleotide nonfluorescently labeled (Probe 1) prepared in 1 and $2 \mu \mathrm{M} 1 \mathrm{X}$ PBS was spotted (40 nL/spot; 4 spots/row) onto both substrates. Once the drops dried, PVDF membranes were exposed to UV light at $365 \mathrm{~nm}$ for 5 minutes, washed with PBS-T, and air-dried. After washing, $25 \mu \mathrm{L}$ of Target $1 *$ (concentrations ranging from 0.0005 to $0.1 \mu \mathrm{M}$, in SSC1x and SSC-T) was spread over the surface with a coverslip. After incubation in a humid chamber for $1 \mathrm{~h}$ at $37^{\circ} \mathrm{C}$, the coverslip was gently removed, and the chip was then washed with PBS-T. The fluorescence intensity of the spots was recorded using the SFR, as mentioned above.

\section{Results and discussion}

\subsection{Surface chemical derivatization}

Derivatization was performed as follows: first, PVDF membranes were immersed in methanol for 15 seconds to remove the air trapped inside the pores, following the instructions of the manufacturer. Then, the membrane was activated by irradiation with a UV lamp at $254 \mathrm{~nm}$, as a promising procedure for the generation of hydroxyl groups onto 
the surface. Different irradiation times were assayed, and the contact angle was measured. As shown in Figure S1, the raw PVDF membranes showed a water contact angle of $130^{\circ}$, whereas contact angles of the activated surfaces lowered up to $83^{\circ}$ after 10 minutes of UV irradiation because of the number of hydroxyl moieties generated on the surface by the oxidation and cleaning treatments. Membranes activated for a longer time did not show a stable contact angle and tended to filtrate quickly, which made us think that a change occurred in the physical structure. For that, an activation time of 10 minutes, which displayed stable contact angles of $83^{\circ}$, was established for accomplishing this process (PVDF-OH). Then, the membranes were again soaked in methanol for 15 seconds to break the barrier between PVDF and the functionalization solution. Immediately, the membranes were functionalized by organosilane chemistry, considering different conditions such as solvents, reaction times, temperatures, and organosilanes. Finally, the membranes were immersed in a $2 \%$ organosilane solution in toluene for 1 hour at $40{ }^{\circ} \mathrm{C}$ to create a thin layer over the surface. Compared to previous studies that used longer procedures $[9,17,24]$, the present methodology required less time.

Two hydrophilic silanes were used for this work: APTES and VTES. APTES silane was chosen as the first functionalization reagent on the basis of the extended work performed by Arafat et al. [17], in which membrane activation by piranha treatment is performed, followed by silanization using the same silane later. In this way, comparison of our activation and functionalization methods can be done in an easy and rapid manner. In addition, primary amine groups allow a quick detection by the ninhydrin test and show characteristic peaks in FT-IR. VTES silane was selected to introduce alkene groups, which are necessary for the later thiol-ene photocoupling reaction. Moreover, hydrophobicity of the PVDF membranes is reduced, thereby allowing a better 
approximation of targets to the anchored probes. Figure 1 shows the layout of the general PVDF modification.

APTES and VTES functionalization slightly decreased the water contact angle of the raw PVDF membranes $\left(119^{\circ}\right.$ and $118^{\circ}$, respectively), thereby enhancing its hydrophilicity. To corroborate the presence of primary amines on the PVDF-APTES membrane, the ninhydrin test was performed. The apparition of a deep blue color in the PVDF-APTES mixture verified the appropriate functionalization of the membrane. As a negative control, a piece of the raw PVDF membrane underwent the same test conditions to modify the membrane; however, no color change occurred [17,35] (Figure 2).

Hence, the activation of PVDF membranes by UV light and the subsequent functionalization by organosilane chemistry constitute an advancement that allows a rapid, simple, mild, and effective procedure to modify PVDF properties.

Thus, to achieve a reproducible protocol to graft surfaces in a homogeneous, nondamaging, and stable way, a broad number of reaction conditions for hydroxylation and silanization processes were studied. A new protocol has been developed in this work, as hydroxyl groups over the membrane are necessary to silanize the substrates, and previously reported methods are aggressive and/or time consuming (alkaline, plasma, and piranha treatments) $[14,15,17-19]$.

\subsection{Characterization of nonmodified and modified membranes}

To establish the chemical configuration of the raw and functionalized membranes, appropriate spectroscopic tools were applied.

\subsubsection{ATR-FTIR}


To detect the presence of new functional groups in the modified PVDF membranes, ATRIR measurements of raw and modified membranes (PVDF-OH, PVDF-APTES, and PVDF-VTES) were made. The spectrum of the raw PVDF membrane displayed the characteristic bands corresponding to this material. With regard to the functional group region, bands related to $\mathrm{C}-\mathrm{H}$ stretching vibrations (symmetric and asymmetric modes) at 3022 and $2981 \mathrm{~cm}^{-1}$ were identified [36]. These stretching bands appeared in all the modified membranes. Remarkably, in comparison to the spectrum of the raw PVDF membrane, the FT-IR spectrum of the PVDF-OH membrane showed the apparition of two additional $\mathrm{C}-\mathrm{H}$ stretching bands located at 2920 and $2852 \mathrm{~cm}^{-1}$. This result, previously reported using piranha activation, [17] is due to the change in the environment of the $\mathrm{CH}_{2}$, in which some neighbors have changed from $\mathrm{CF}_{2}$ to $\mathrm{CHOH}$ motifs as a result of UV light treatment; thus, a red shift is expected to occur. In addition, for functionalized membranes (PVDF-APTES and PVDF-VTES), new signals in the range of 3000 to 2800 were detected. In the case of PVDF-APTES, the new generated $\mathrm{CH}_{2}$ mode of vibration appeared at 2930 and $2876 \mathrm{~cm}^{-1}$ with higher intensity and minor shift, which might be due to the contribution of the propyl group [17]. For PVDF-VTES, two new stretching vibrations situated at 2912 and $2841 \mathrm{~cm}^{-1}$ were recognized. In addition, the spectrum of the PVDF-APTES membrane displayed two other new significant features related to primary amine groups. First, two characteristic peaks for the amine group can be clearly detected at 3349 and $3287 \mathrm{~cm}^{-1}$, which represent typical primary amine stretching vibrations (Figure 3). Second, two additional peaks related to bending vibrations of primary amines at 1574 and $1477 \mathrm{~cm}^{-1}$ were observed (Figure S2). Figure S3 displays the fingerprint region (from 1500 to $400 \mathrm{~cm}^{-1}$ ) of nonmodified and modified membranes. FTIR spectra in this region followed a highly similar profile for all the membranes. 


\subsubsection{XPS}

XPS spectra of raw PVDF and PVDF-APTES membranes were also recorded. The spectrum of the PVDF-APTES membrane showed new peaks at 398, 157, and $102 \mathrm{eV}$ related to the binding energies of $\mathrm{N} 1 \mathrm{~s}, \mathrm{Si} 2 \mathrm{~s}$, and $\mathrm{Si} 2 \mathrm{p}$. These results corroborated the presence of silicon $(\mathrm{Si})$ and nitrogen $(\mathrm{N})$ motifs in the modified membrane, which demonstrated the successful functionalization. The intensity of the $\mathrm{O} 1 \mathrm{~s}$ band $(531 \mathrm{eV})$ was also increased because of the silanol groups generated onto the surface (Figure 4). In addition, quantification of peak $\mathrm{C} 1 \mathrm{~s}$ contributions revealed an increase in the signals due to the $\mathrm{C}-\mathrm{C}$ band $(284.8 \mathrm{eV})$, which proved the presence of propyl groups of the organosilane, and a decrease in $\mathrm{C}-\mathrm{F}$ bond contribution $(289.4 \mathrm{eV})$ due to the dehydrofluorination process [37]. (Figure S4, ESI).

\subsection{Microarray bioassays}

The demonstration of the derivatization methodology of PVDF membranes by simple UV irradiation and silanization and its availability at a mass scale made us consider its use as a support for carrying out biological recognition assays to endow membranes for new applications. As a proof of concept, raw PVDF and PVDF-VTES membranes were applied for microarray technology. Hence, immobilization of nucleic acids and hybridization assays were performed with both membranes, and results were compared between the two assays and also with the results of a previous work [21].

\subsubsection{Nucleic acid tethering}

Here, we compare the immobilizations of thiolated probes onto the raw PVDF membrane and the PVDF-VTES membrane. The novel fluor-thiol photocoupling reaction allowed 
the covalent anchoring of thiolated probes onto the $\mathrm{C}-\mathrm{F}$ bonds of the raw PVDF membrane [21], whereas the well-known thiol-ene photocoupling reaction allowed fixing of the probes to the alkene motifs generated on the membrane [10-12,15].

Thus, membranes were spotted with a thiolated probe that is fluorescent tag (Probe $1 *$ ) in 1 and $2 \mu \mathrm{M} 1 \mathrm{X}$ PBS. After the drops were dried, irradiated with UV light at $365 \mathrm{~nm}$ for 5 minutes, and washed with PBS-T, the fluorescence of the microarray was recorded using the SFR. Both membranes immobilized the thiolated nucleic acids successfully; however, the PVDF-VTES membrane showed a higher immobilization density (approximately twofold) than the raw PVDF membrane. As negative controls, two experiments were performed: First, the irradiation step was skipped to corroborate the use of UV light to immobilize the thiolated probe (Probe $1^{*}$ ). Second, a nonthiolated probe (Target $1^{*}$ ) was used to confirm the presence of the thiol group. Figure 5 displays both immobilization and negative control experiments.

Immobilization studies were performed on both membrane sides. Although successful immobilizations were demonstrated on both sides, the bottom side displayed a better consistency and homogeneity of the spots (Figure S5). Therefore, further experiments were performed on the bottom side of the PVDF membranes.

\subsubsection{Target detection}

Hybridization with the complementary target was performed in raw PVDF and PVDFVTES membranes. Thus, nonlabeled thiolated probe (Probe 1) in 1 and $2 \mu \mathrm{M} 1 \mathrm{X}$ PBS was spotted onto both surfaces, and immobilization was carried out in the same way as that before. After washing with PBS-T and drying, hybridization was carried out with a fully complementary AlexaFluor647-labeled probe (Target $1 *$ ) at different concentrations (from 0.0005 to $0.1 \mu \mathrm{M}$ in $1 \mathrm{X}$ SSC) in a humidity chamber. The microarrays were 
incubated for $1 \mathrm{~h}$ at $37^{\circ} \mathrm{C}$ and then washed; the fluorescence intensity of the spots was recorded using the SFR. Owing to the high hydrophobicity of the raw PVDF membrane, the contact between the solution and the membrane surface was hindered; thus, a high amount of target (up to $0.1 \mu \mathrm{M}$ ) was necessary to achieve a detectable fluorescence signal (Figure 6).

To solve this problem, SDS or Tween 20 surfactant was added to the target solution in the hybridization step to reduce the hydrophobic interactions and favor the approximation of the target to the anchored probe. Using a small amount of the detergent $(0.5 \%)$ in the recognition process, up to $5 \mathrm{nM}$ of Target $1^{*}$ was detected using the PVDF-VTES membrane as the substrate, whereas with the raw PVDF membrane, up to $1 \mathrm{nM}$ was detected (Figure 7). The sensitivity of our microarray was limited by the sensitivity of the homemade fluorescence reader, SFR, which is within a detection limit of 1 and $5 \mathrm{nM}$, independent of the system and surface used. Considering the limitations of the reading device, both platforms revealed great suitability for the recognition of very low levels of nucleic acid sequences, and changing to other more sensitive detection devices or using colorimetric or enzymatic detection can lead to limits of detection in the range of $\mathrm{pM}$, as we previously demonstrated $[22,23]$. While the raw PVDF membrane showed slightly better limit of detection, the PVDF-VTES membranes could function without the need for surfactants and permit other methods of anchoring apart from thiol-ene coupling, i.e., alkene or acrylate polymerization.

Finally, if we compare the performance of the raw PVDF membranes with recently reported perfluorinated glass slides [21], in which $0.5 \mathrm{nM}$ of the target was detected, a slightly lower detection capacity was presented by this novel material. However, the PVDF membrane did not require previous modification steps for the probe immobilization and hybridization processes, as in the case of functionalized glass slides, 
thus saving reagents and time. Hence, PVDF membranes allowed a quicker detection evaluation, which affords an encouraging substrate for microarray technologies. Furthermore, the flexibility of the membranes permits an easier integration and applicability to other fields.

\section{Conclusions}

An alternative method for activating PVDF flat sheet membranes is shown in this study. The method is milder and easier than previous methods, as it uses only light for activation. The membranes activated in such way undergo silanization rapidly and as easily as membranes activated by other harsher methods.

As a proof of concept of its applicability, PVDF and PVDF-VTES membranes were used as substrates for microarray technology. For that, immobilization of thiolated nucleic acids and later hybridization with the complementary strand were carried out. The functionalized PVDF-VTES membrane displayed higher immobilization densities than the raw PVDF membranes; however, detection capacity was not improved. The raw PVDF membrane was had a detection of up to $1 \mathrm{nM}$ of complementary strand by adding SDS surfactant to the target solution. This detection capacity is very close to the reported value using perfluorinated glass slides as supports and avoids the tedious previous functionalization process, thus saving reagents and time, without almost loss of sensitivity.

Finally, these advances pave the way to the biofunctionalization of raw PVDF membranes with other bioreceptors such as antibodies and the direct functionalization using thiolated organic compounds taking the advantage of UV irradiation. The second approximation would allow the modification of surface properties in a rapid, easy, and effective way without using organosilanes. 


\section{Acknowledgments}

Financial support from BIHOLOG (Project CTQ2016-75749-R) and FEDER is acknowledged. P.J.-M. acknowledges the Spanish Ministry of Economy, Industry and Competitiveness for the public FPI grant (Project CTQ2013-45875-R) and cofinancing by the European Social Fund.

Declarations of interest: None.

\section{Bibliography}

[1] F. Liu, N.A. Hashim, Y. Liu, M.R.M. Abed, K. Li, Progress in the production and modification of PVDF membranes, J. Membr. Sci. 375 (2011) 1-27.

[2] G. dong Kang, Y. ming Cao, Application and modification of poly(vinylidene fluoride) (PVDF) membranes - A review, J. Membr. Sci. 463 (2014) 145-165.

[3] Y. Sui, Z. Wang, X. Gao, C. Gao, Antifouling PVDF ultrafiltration membranes incorporating PVDF-g-PHEMA additive via atom transfer radical graft polymerizations, J. Membr. Sci. 413-414 (2012) 38-47.

[4] B. Onal-Ulusoy, Effects of Plasma-Modified Polyvinylidenefluoride and Polyethersulfone Ultrafiltration (UF) Membrane Treatments on Quality of Soybean Oil, J. Food Qual. 38 (2015) 285-296.

[5] M.F. Rabuni, N.M. Nik Sulaiman, M.K. Aroua, N.A. Hashim, Effects of alkaline environments at mild conditions on the stability of PVDF membrane: An experimental study, Ind. Eng. Chem. Res. 52 (2013) 15874-15882.

[6] X. Shen, X. Yin, Y. Zhao, L. Chen, Improved protein fouling resistance of PVDF membrane grafted with the polyampholyte layers, Colloid Polym. Sci. 293 (2015) $1205-1213$. 
[7] S.-H. Lin, K.-L. Tung, H.-W. Chang, K.-R. Lee, Influence of fluorocarbon flatmembrane hydrophobicity on carbon dioxide recovery, Chemosphere. 75 (2009) $1410-1416$.

[8] X.X. Shen, W.F. Yang, Q.F. Zhang, Hydrophobic Modification of PVDF, in: Funct. Electron. Mater. IUMRS-ICA2010, Trans Tech Publications, 2011: pp. 658-661..

[9] X. Yang, R. Wang, L. Shi, A.G. Fane, M. Debowski, Performance improvement of PVDF hollow fiber-based membrane distillation process, J. Membr. Sci. 369 (2011) $437-447$.

[10] S. Sairiam, C.H. Loh, R. Wang, R. Jiraratananon, Surface modification of PVDF hollow fiber membrane to enhance hydrophobicity using organosilanes, J. Appl. Polym. Sci. 130 (2013) 610-621.

[11] S. Hao, Y. Geng, Z. Jia, UV pre-activation/thermal initiated grafting of caffeic acid on PVDF for preparation of adsorptive membranes for cesium, React. Funct. Polym. 132 (2018) 120-126.

[12] Y. He, X. Chen, F. Dai, R. Xu, N. Yang, X. Feng, Y. Zhao, L. Chen, Immobilization of poly(N-acryoyl morpholine) via hydrogen-bonded interactions for improved separation and antifouling properties of poly(vinylidene fluoride) membranes, React. Funct. Polym. 123 (2018) 80-90.

[13] J. Zhao, H. Han, Q. Wang, C. Yan, D. Li, J. Yang, X. Feng, N. Yang, Y. Zhao, L. Chen, Hydrophilic and anti-fouling PVDF blend ultrafiltration membranes using polyacryloylmorpholine-based triblock copolymers as amphiphilic modifiers, React. Funct. Polym. (2019).

[14] G.J. Ross, J.. Watts, M.. Hill, P. Morrissey, Surface modification of poly(vinylidene fluoride) by alkaline treatment Part 2. Process modification by the use of phase transfer catalysts, Polymer. 42 (2001) 403-413. 
[15] G.J. Ross, J.F. Watts, M.P. Hill, P. Morrissey, Surface modification of poly(vinylidene fluoride) by alkaline treatment 1 . The degradation mechanism, Polymer. 41 (2000) 1685-1696.

[16] H. Dong, K. Xiao, X. Tang, Z. Zhang, J. Dai, R. Long, W. Liao, Preparation and characterization of polyurethane (PU)/polyvinylidene fluoride (PVDF) blending membrane, Desalination Water Treat. 57 (2016) 3405-3413.

[17] S. Al-Gharabli, J. Kujawa, M.O. Mavukkandy, H.A. Arafat, Functional groups docking on PVDF membranes: Novel Piranha approach, Eur. Polym. J. 96 (2017) $414-428$.

[18] Y. Chen, H. Kim, Preparation of superhydrophobic membranes by electrospinning of fluorinated silane functionalized poly(vinylidene fluoride), Appl. Surf. Sci. 255 (2009) 7073-7077.

[19] W.-T. Xu, Z.-P. Zhao, M. Liu, K.-C. Chen, Morphological and hydrophobic modifications of PVDF flat membrane with silane coupling agent grafting via plasma flow for VMD of ethanol-water mixture, J. Membr. Sci. 491 (2015) 110120.

[20] G. Botelho, M.M. Silva, A.M. Gonçalves, V. Sencadas, J. Serrado-Nunes, S. Lanceros-Mendez, Performance of electroactive poly(vinylidene fluoride) against UV radiation, Polym. Test. 27 (2008) 818-822.

[21] P. Jiménez-Meneses, M.-J. Bañuls, R. Puchades, Á. Maquieira, Fluor-thiol Photocoupling Reaction for Developing High Performance Nucleic Acid (NA) Microarrays, Anal. Chem. 90 (2018) 11224-11231.

[22] M.-J. Bañuls, P. Jiménez-Meneses, A. Meyer, J.-J. Vasseur, F. Morvan, J. Escorihuela, R. Puchades, A. Maquieira, Improved performance of DNA microarray 
multiplex hybridization using probes anchored at several points by Thiol-Ene or Thiol-Yne click chemistry, Bioconjug. Chem. 28 (2017) 496-506.

[23] R. Alonso, P. Jiménez-Meneses, J. García-Rupérez, M.-J. Bañuls, Á. Maquieira, Thiol-ene click chemistry towards easy microarraying of half-antibodies, Chem. Commun. 54 (2018) 6144-6147.

[24] Z. Zheng, Z. Gu, R. Huo, Z. Luo, Superhydrophobic poly(vinylidene fluoride) film fabricated by alkali treatment enhancing chemical bath deposition, Appl. Surf. Sci. 256 (2010) 2061-2065.

[25] P. Angenendt, J. Glökler, D. Murphy, H. Lehrach, D.J. Cahill, Toward optimized antibody microarrays: a comparison of current microarray support materials, Anal. Biochem. 309 (2002) 253-260.

[26] P. Angenendt, Progress in protein and antibody microarray technology, Drug Discov. Today. 10 (2005) 503-511.

[27] S. Hu, Z. Xie, J. Qian, S. Blackshaw, H. Zhu, Functional protein microarray technology: Functional protein microarray technology, Wiley Interdiscip. Rev. Syst. Biol. Med. 3 (2011) 255-268.

[28] F.X.R. Sutandy, J. Qian, C.-S. Chen, H. Zhu, Overview of Protein Microarrays, Curr. Protoc. Protein Sci. 72 (2013) 27.1.1-27.1.16.

[29] K. Bussow, A method for global protein expression and antibody screening on highdensity filters of an arrayed cDNA library, Nucleic Acids Res. 26 (1998) 50075008 .

[30] A. Lueking, M. Horn, H. Eickhoff, K. Büssow, H. Lehrach, G. Walter, Protein Microarrays for Gene Expression and Antibody Screening, Anal. Biochem. 270 (1999) 103-111. 
[31] J. Escorihuela, M.J. Bañuls, S. Grijalvo, R. Eritja, R. Puchades, Á. Maquieira, Direct covalent attachment of DNA microarrays by rapid thiol-ene "click" chemistry, Bioconjug. Chem. 25 (2014) 618-627.

[32] J. Escorihuela, M.-J. Bañuls, R. Puchades, Á. Maquieira, Site-specific immobilization of DNA on silicon surfaces by using the thiol-yne reaction, J Mater Chem B. 2 (2014) 8510-8517.

[33] J. Escorihuela, M.J. Bañuls, R. Puchades, Á. Maquieira, DNA microarrays on silicon surfaces through thiol-ene chemistry., Chem. Commun. 48 (2012) 21162118.

[34] D. Mira, R. Llorente, S. Morais, R. Puchades, A. Maquieira, J. Marti, High throughput screening of surface-enhanced fluorescence on industrial standard digital recording media, Proc SPIE. 5617 (2004) 364-373.

[35] E. Kaiser, R.L. Colescott, C.D. Bossinger, P.I. Cook, Color test for detection of free terminal amino groups in the solid-phase synthesis of peptides, Anal. Biochem. 34 (1970) 595-598.

[36] H. Bai, X. Wang, Y. Zhou, L. Zhang, Preparation and characterization of poly(vinylidene fluoride) composite membranes blended with nano-crystalline cellulose, Prog. Nat. Sci. Mater. Int. 22 (2012) 250-257.

[37] N. Akashi, S. Kuroda, Protein immobilization onto poly (vinylidene fluoride) microporous membranes activated by the atmospheric pressure low temperature plasma, Polymer. 55 (2014) 2780-2791. 


\section{Figure captions}

Figure 1. Layout of the simple activation and functionalization processes of PVDF membranes.

Figure 2. Ninhydrin test performed for a functionalized PVDF-APTES membrane (left) and a raw PVDF membrane (right). As can be seen, blue color indicating the presence of amines was developed only by the PVDF-APTES membrane.

Figure 3. Comparison of ATR-IR spectra of nonmodified and modified PVDF membranes. Additional functional groups generated on the membranes displayed new peaks in the spectra. Blue: Raw PVDF membrane presents two $\mathrm{C}-\mathrm{H}$ stretching vibrations, characteristic of this material (3022 and $2981 \mathrm{~cm}^{-1}$ ). Yellow: PVDF-OH membrane reveals two additional peaks at 2920 and $2952 \mathrm{~cm}^{-1}$, which corresponds to new C-H stretching bands and a broad band related to the hydroxyl groups. Green: PVDF-VTES membrane shows the emergence of $\mathrm{CH}_{2}$ vibrations at 2912 and $2941 \mathrm{~cm}^{-1}$. Orange: PVDF-APTES membrane displays two peaks related to primary amines (3356 and 3288 $\mathrm{cm}^{-1}$ ) and two additional peaks related to $\mathrm{C}-\mathrm{H}$ stretching vibrations at 2930 and 2876 $\mathrm{cm}^{-1}$.

Figure 4. XPS spectra comparison between the raw PVDF membrane and PVDF membrane functionalized with APTES. Apparition of new peaks in the functionalized membranes (PVDF-APTES) at 398, 157, and $102 \mathrm{eV}$, revealed the presence of N1s, Si2s, and $\mathrm{Si} 2 \mathrm{p}$ motifs, respectively. Increase in the $\mathrm{O} 1 \mathrm{~s}$ intensity $(531 \mathrm{eV})$ was appreciated.

Figure 5. Top. Maximal immobilization densities of raw PVDF and PVDF-VTES membranes. Fluorescence intensities before and after washing were compared to calculate these values. Bottom. Negative controls corroborated the needed of irradiation and thiol group to undertake the fluor-thiol photocoupling reaction. 
Figure 6. Raw PVDF membranes showed a low sensitivity detection in the hybridization process using 1X SSC as a hybridization buffer. Minimal concentration of target to detect worthy fluorescence signal was $0.1 \mu \mathrm{M}$.

Figure 7. Hybridization assays with decreasing concentrations of Target $1^{*}$, using PVDF and PVDF-VTES membranes as substrates, were performed. Addition of SDS as a surfactant provided successful recognitions. The image shows the neat fluorescence intensity versus concentration of Target $1 *$ used in the hybridization process. Detection limits are $1 \mathrm{nM}$ and $5 \mathrm{nM}$ for PVDF and PVDF-VTES membranes, respectively. 


\section{Figures and tables}

Figure 1

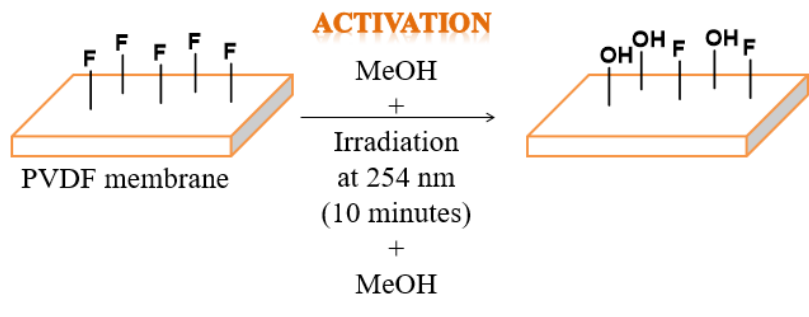

FUNCTIONALIZATION

$2 \%$ organosilane in toluene for 1 hour at $40^{\circ} \mathrm{C}$
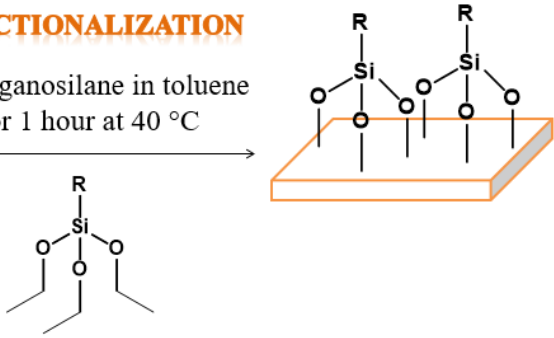

Organosilane $=$ APTES or VTES 
Figure 2

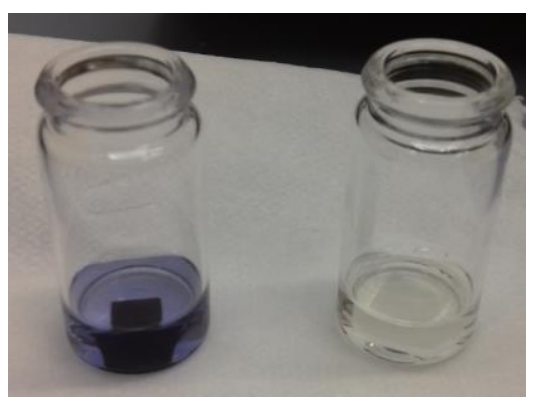


Figure 3

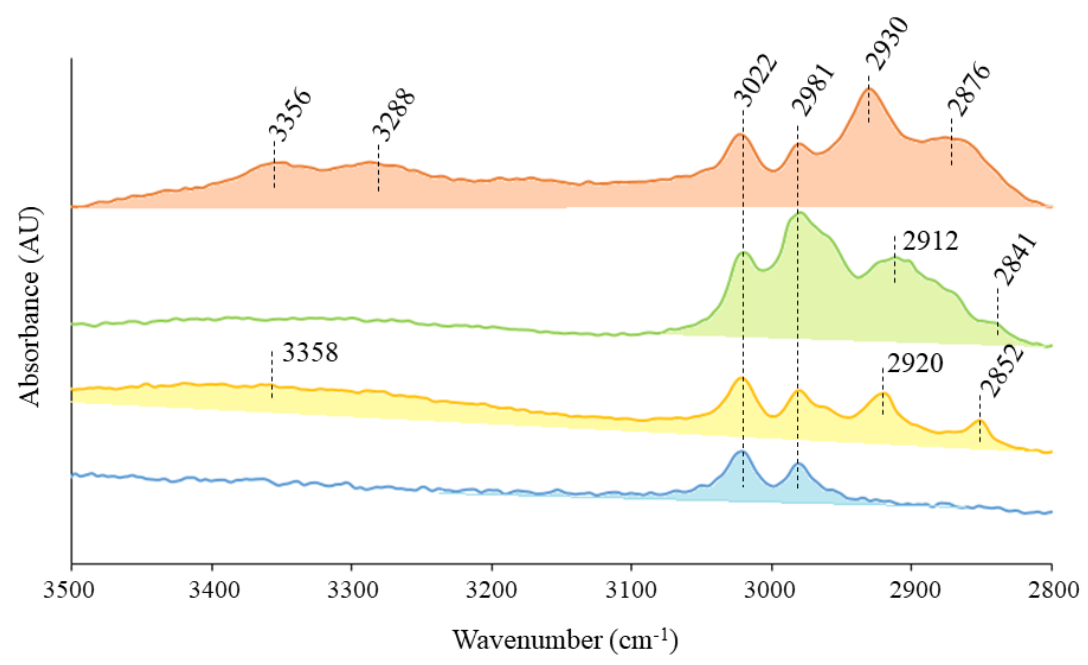


Figure 4

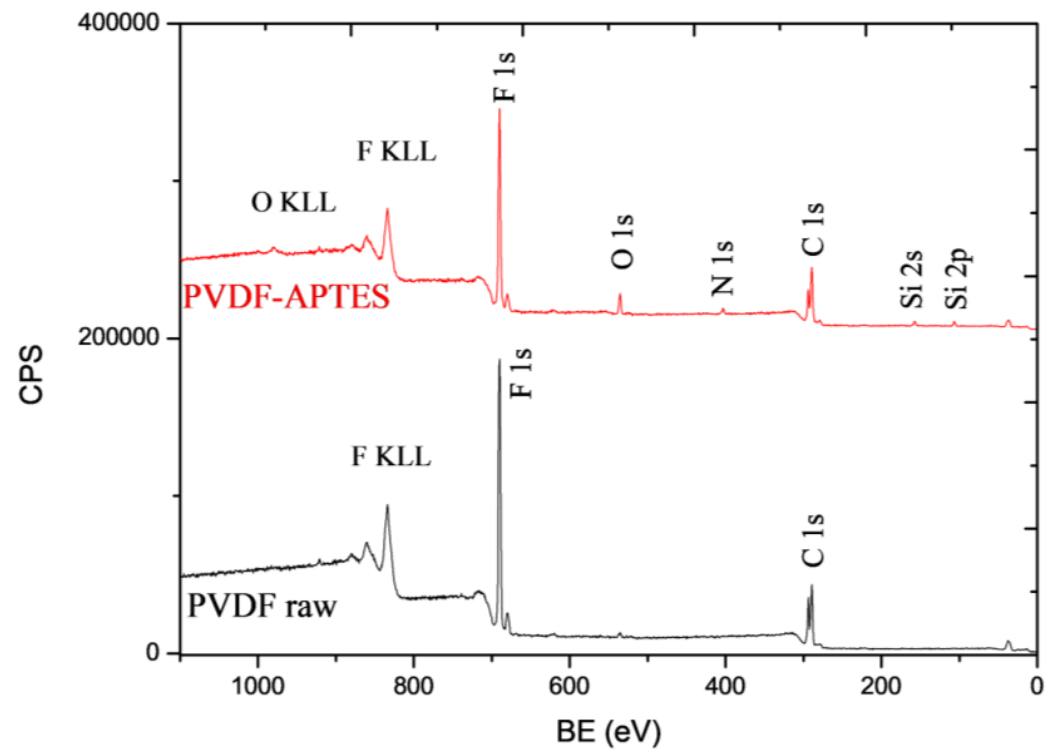


Figure 5

\begin{tabular}{|c|c|c|c|}
\hline \multirow{3}{*}{ Probe $1^{*}(1 \mu \mathrm{M})$} & tion + washing & \multicolumn{2}{|c|}{ Irradiation + washing } \\
\hline & PVDF & PVDF-Vinyl & $\mathrm{HS} \sim$ \\
\hline & 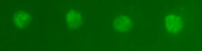 & 000 & Probe $1^{*}(1 \mu \mathrm{M})$ \\
\hline \multirow[t]{2}{*}{ Probe $1 *(2 \mu \mathrm{M})$} & $00 \% 0$ & 000 & Probe $1^{*}(2 \mu \mathrm{M})$ \\
\hline & $=5 \mathrm{pmol} / \mathrm{cm}^{2}$ & $\delta=11 \mathrm{pmol} / \mathrm{cm}^{2}$ & \\
\hline Probe $1 *(1 \mu \mathrm{M})$ & & & Target $1^{*}(1 \mu \mathrm{M})$ \\
\hline Probe $1 *(2 \mu \mathrm{M})$ & & . & Target $1 *(2 \mu \mathrm{M})$ \\
\hline${ }_{\mathrm{HS}} \sim \boldsymbol{M}_{\mathrm{Cy} 5}$ & PVDF & PVDF & ヘn \\
\hline \multicolumn{2}{|c|}{ Non irradiation + washing } & Irradiation $+v$ & shing \\
\hline
\end{tabular}


Figure 6

$$
\text { PVDF }
$$

Target $1 *(0.05 \mu \mathrm{M})$ Target $1 *(0.1 \mu \mathrm{M})$

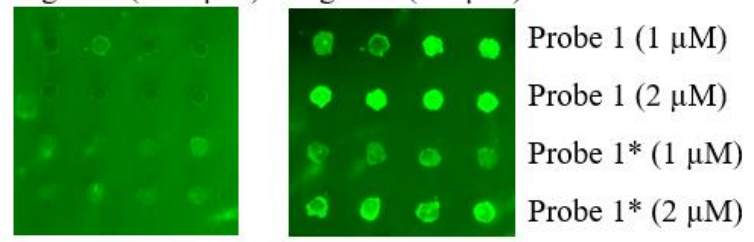


Figure 7

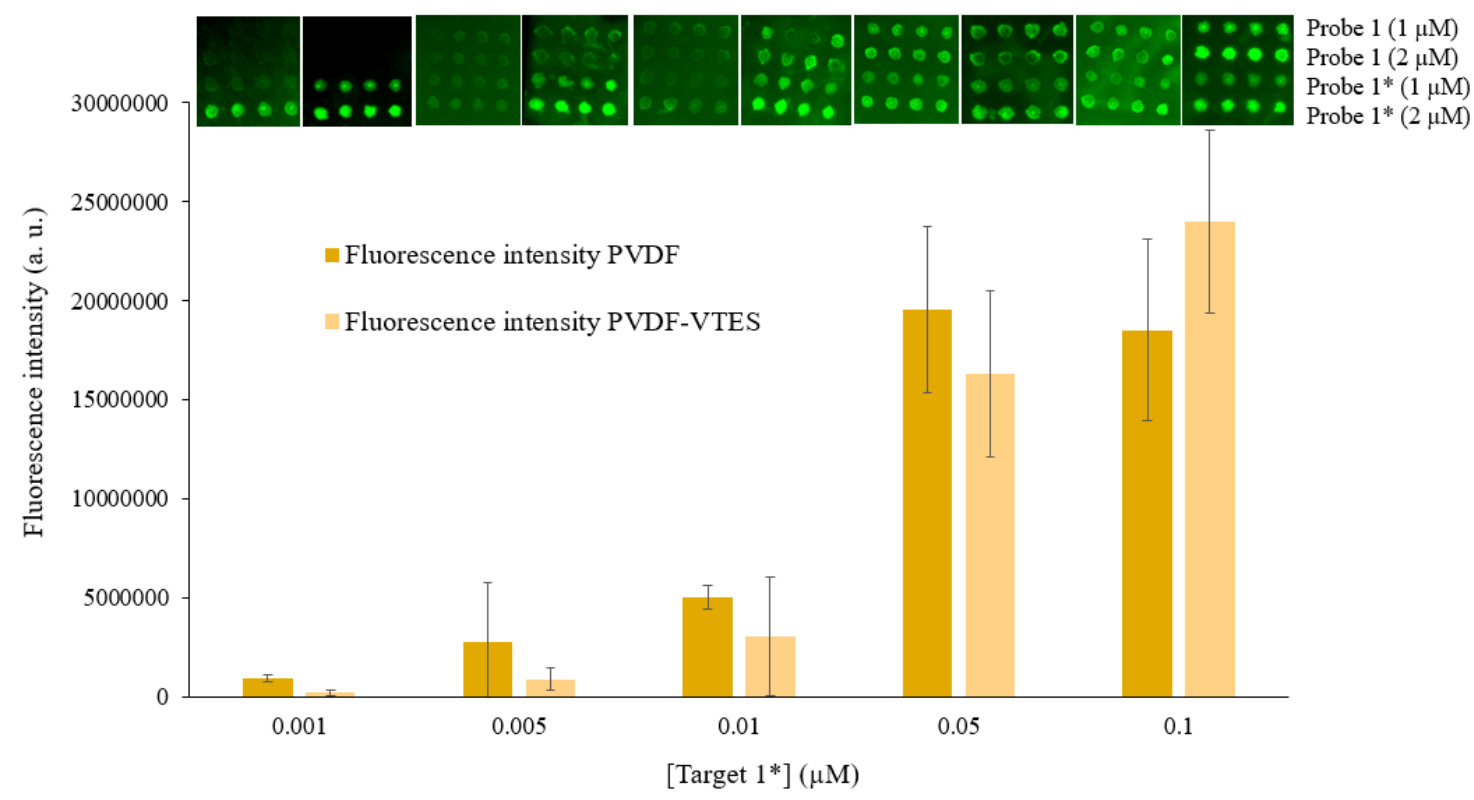


Table 1. Oligonucleotide sequences used

\begin{tabular}{|l|l|l|l|}
\hline Name & Sequence $\left(5^{\prime}\right.$ to $\left.3^{\prime}\right)$ & $5^{\prime}$ & $3^{\prime}$ \\
\hline Probe $1^{*}$ & $(\mathrm{~T})_{15}$-CCCGATTGACCAGCTAGCATT & SH & Cy5 \\
\hline Probe 1 & $(\mathrm{T})_{15}$-CCCGATTGACCAGCTAGCATT & SH & - \\
\hline Target $1^{*}$ & AATGCTAGCTGGTCAATCGGG & $\mathrm{Ax}^{\mathrm{a}}$ & - \\
\hline
\end{tabular}

a Alexa Fluor® 647 


\section{Supplementary information}

Novel and rapid activation of polyvinylidene fluoride membranes by UV light

Pilar Jiménez-Meneses, María-José Bañuls*, Rosa Puchades, Ángel Maquieira

\begin{tabular}{|c|c|c|c|c|c|c|}
\hline PVDF & $\begin{array}{l}\text { Nonactivate } \\
\text { d }\end{array}$ & $\begin{array}{l}\text { Irradiated } \\
\text { for } 30 \\
\text { seconds }\end{array}$ & $\begin{array}{l}\text { Irradiated } \\
\text { for } 2 \\
\text { minutes }\end{array}$ & $\begin{array}{l}\text { Irradiated } \\
\text { for } 5 \\
\text { minutes }\end{array}$ & $\begin{array}{l}\text { Irradiated } \\
\text { for } 7 \\
\text { minutes }\end{array}$ & $\begin{array}{l}\text { Irradiated } \\
\text { for } 10 \\
\text { minutes } \\
\end{array}$ \\
\hline WCA & $130^{\circ}$ & $130^{\circ}$ & $122^{\circ}$ & $121^{\circ}$ & $100^{\circ}$ & $83^{\circ}$ \\
\hline 140 & 130 & 130 & & & \multirow[b]{3}{*}{100} & \multirow{5}{*}{83} \\
\hline 120 & & 尼 & 122 & 121 & & \\
\hline 100 & & & & & & \\
\hline & & & & & & \\
\hline 80 & & 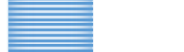 & 㡡 & 套 & 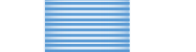 & \\
\hline 60 & 占 & 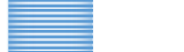 & 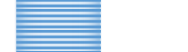 & 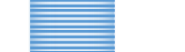 & $\underline{\underline{\underline{|l|}}}$ & 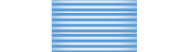 \\
\hline 40 & 咅 & 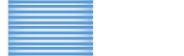 & $\underline{\underline{\underline{\underline{|l|}}}}$ & 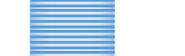 & 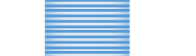 & 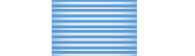 \\
\hline 20 & & 兒 & 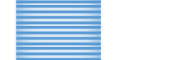 & 套 & 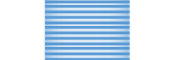 & 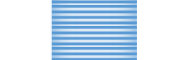 \\
\hline \multirow{2}{*}{0} & & 㪯 & 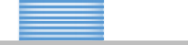 & 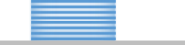 & & 呈 \\
\hline & Ton-activated & $\begin{array}{l}\text { adiated } 30 \\
\text { seconds }\end{array}$ & $\begin{array}{l}\text { radiated } 2 \\
\text { minutes }\end{array}$ & $\begin{array}{l}\text { rradiated } 5 \\
\text { minutes }\end{array}$ & $\begin{array}{c}\text { Irradiated } 7 \\
\text { minutes }\end{array}$ & $\begin{array}{c}\text { Irradiated } 10 \\
\text { minutes }\end{array}$ \\
\hline
\end{tabular}

Figure S1. Water contact angles displayed for PVDF membranes after different activation times. 


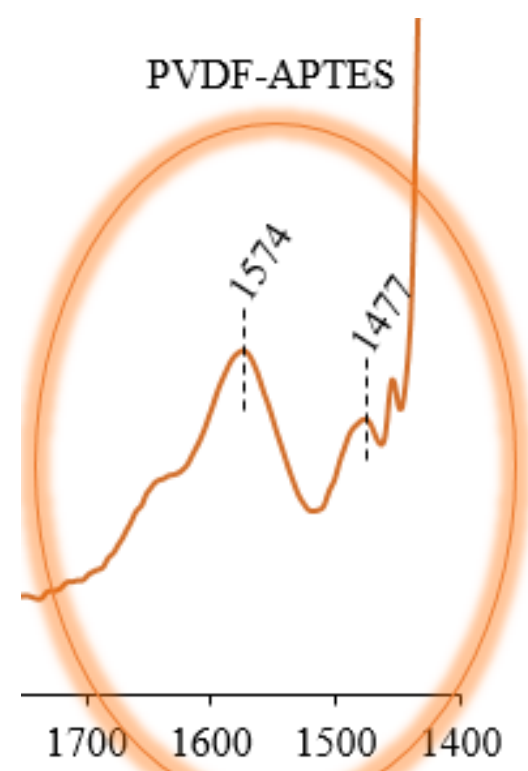

Figure S2. ATR-IR spectra (approximately $1700-1400 \mathrm{~cm}^{-1}$ ) of the PVDF-APTES membrane. Bending vibrations corresponding to primary amines, at 1574 and $1477 \mathrm{~cm}^{-1}$, were recorded.

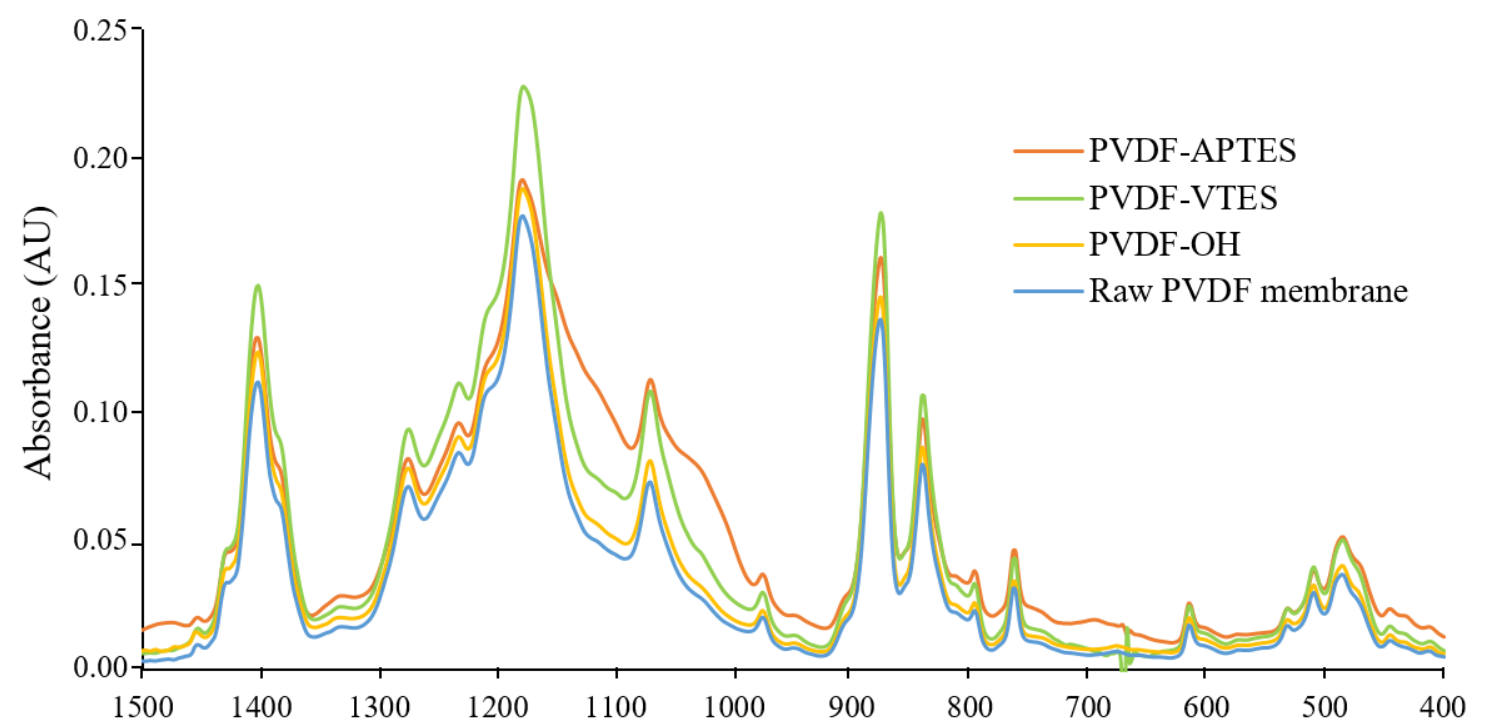

Figure S3. ATR-IR spectra $\left(1500-400 \mathrm{~cm}^{-1}\right)$ of the fingerprint region of the raw PVDF membrane, activated PVDF membrane, and functionalized PVDF-APTES and -VTES. A similar profile is observed. 

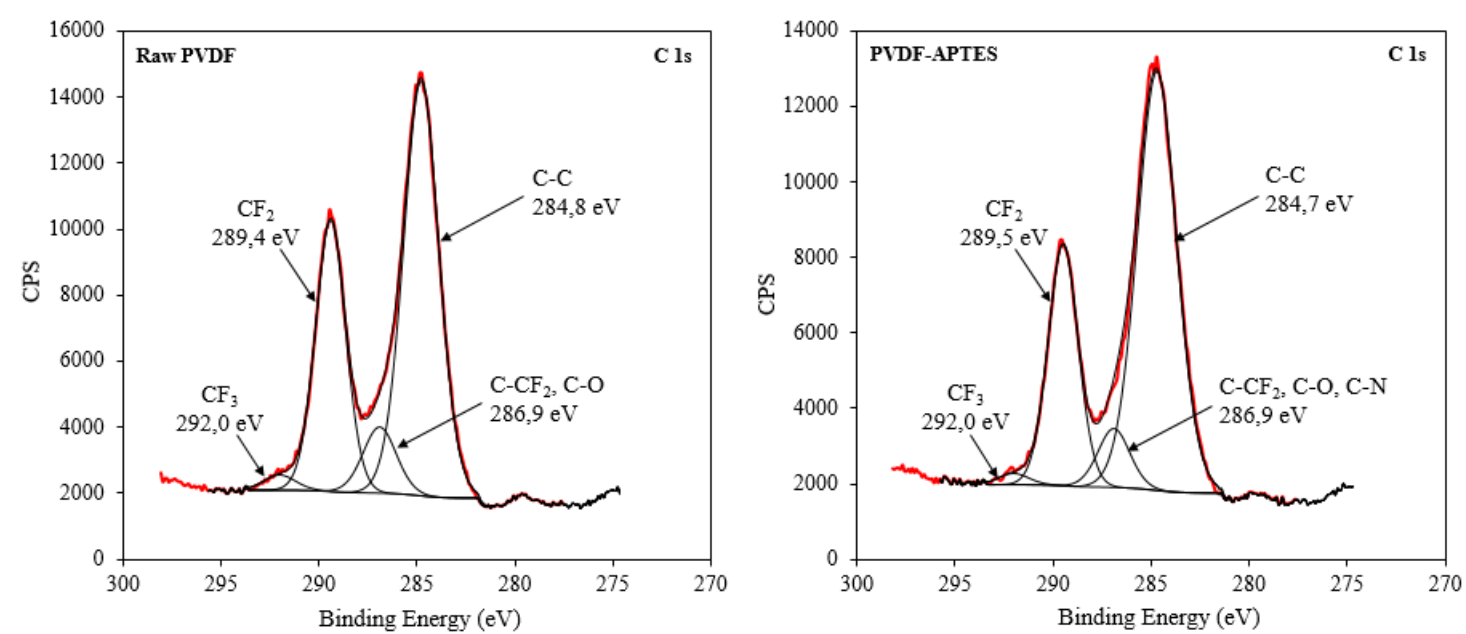

\begin{tabular}{|c|cccc|}
\hline Sample & \multicolumn{4}{|c|}{ Raw PVDF membrane } \\
\hline Name & $\mathrm{C}-\mathrm{C}$ & $\mathrm{C}-\mathrm{CF}_{2}, \mathrm{C}-\mathrm{O}$ & $\mathrm{CF}_{2}$ & $\mathrm{CF}_{3}$ \\
\hline BE (eV) & 284.8 & 286.9 & 289.4 & 292.0 \\
\hline$\%$ At & 57.3 & 8.8 & 32.0 & 1.9 \\
\hline
\end{tabular}

\begin{tabular}{|c|cccc|}
\hline Sample & \multicolumn{4}{|c|}{ PVDF-APTES membrane } \\
\hline Name & $\mathrm{C}-\mathrm{C}$ & $\mathrm{C}-\mathrm{CF}_{2}, \mathrm{C}-\mathrm{O}, \mathrm{C}-\mathrm{N}$ & $\mathrm{CF}_{2}$ & $\mathrm{CF}_{3}$ \\
\hline $\mathrm{BE}(\mathrm{eV})$ & 284.7 & 286.9 & 289.5 & 292.0 \\
\hline$\%$ At & 64.0 & 7.1 & 27.6 & 1.3 \\
\hline
\end{tabular}

Figure S4. XPS experiments displayed the contributions of $\mathrm{C}-\mathrm{C}$ and $\mathrm{CF}_{2}$ to the $\mathrm{C} 1 \mathrm{~s}$ binding energy for PVDF and PVDF-APTES membranes. For functionalized membranes, the amount of $\mathrm{C}-\mathrm{C}$ groups $(284.8 \mathrm{eV})$ increased, whereas the percentage of $\mathrm{CF}_{2}(289.4$ $\mathrm{eV}$ ) decreased, which corroborates the functionalization of the membrane.
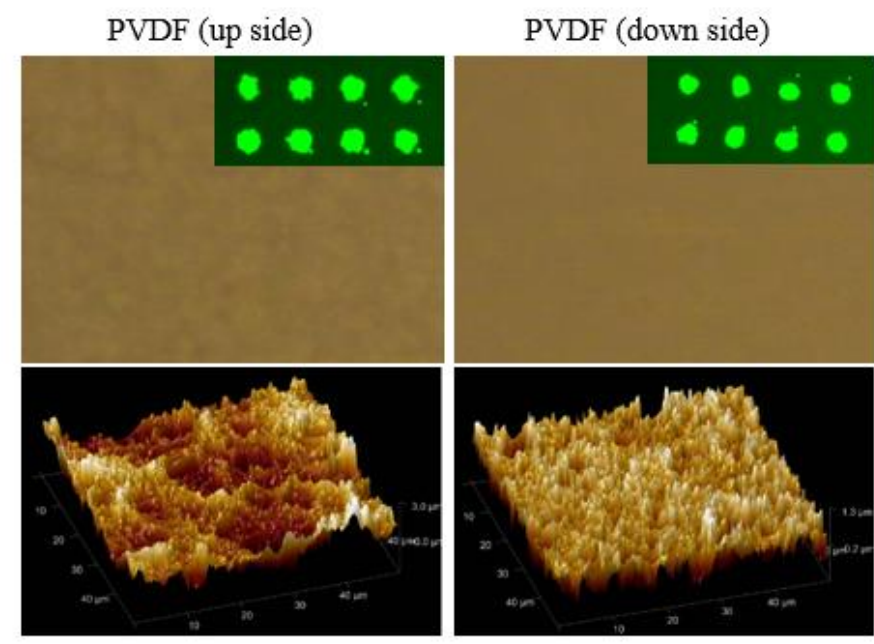

Figure S5. Comparison between the top and bottom sides of the PVDF membranes. Top.

Microscope pictures and array image. Bottom. AFM measurements. 